\title{
MAYABEQUE, UNA NUEVA PROVINCIA EN CUBA: ESTUDIO DE CASO SOBRE LA CONSTRUCCIÓN DE LA IDENTIDAD REGIONAL
}

\author{
MAYABEQUE, THE NEW PROVINCE IN CUBA: STUDY CASE \\ ABOUT THE BUILDING UP A NEW REGIONAL IDENTITY
}

\author{
Elier González-Martínez
}

Cubano. Profesor universitario. Universidad Politécnica Salesiana de Ecuador.

Email: egonzalez@ups.edu.ec

\begin{tabular}{|l|l}
\hline Recibido: 24.09 .18 & Aprobado: 05.07.19 \\
\hline
\end{tabular}

DOI:10.15517/isucr.v20i41.38808

\section{Resumen}

La provincia Mayabeque en Cuba nació de la división administrativa de La Habana, decisión aplicada verticalmente por el Estado cubano. En el proceso de división territorial se olvidaron fenómenos culturales como su gran diversidad cultural y el anclaje a una identidad territorial formada por siglos de memoria e historia regional. El objetivo de la investigación fue evidenciar la construcción de la nueva identidad territorial "mayabequense" en el discurso mediático a raíz de esta división. Partiendo de la teoría de Bayart (1996) "la invención de la tradición" se realizó el análisis de contenido de la prensa escrita regional durante el primer año de existencia de la provincia. Los resultados evidencian que la identidad territorial fue construida como una forma de improvisación basada en la rememoración y resignificación de las identidades locales.

Palabras clave: identidad; construcción regional; cultura; división administrativa; Cuba;

\begin{abstract}
The Mayabeque province in Cuba was established by the administrative division of Havana, a decision applied vertically by the Cuban State. In the process of territorial division cultural phenomena were forgotten, such as its broad identity diversity and the anchorage to a territorial identity formed by centuries of regional memory and history. The objective of the investigation is to demonstrate the construction of the new territorial Mayabequense identity in the media discourse as a result of this division. Based on the theory of Bayart (1996) "the invention of tradition" the content analysis of the regional written press was carried out during the first year of the province's existence. The results show that the territorial identity was conceived as a kind of improvisation based on the remembrance and resignifying of local identities.
\end{abstract}

Keywords: identity; regional construction; culture; administrative division; Cuba; 


\section{Introducción}

El presente estudio se suscribe a Cuba y al analizar su división político-administrativa se constata que la organización territorial es por provincias, municipios, y el territorio especial Isla de la Juventud, pequeña isla fuera del gran archipiélago; en cifras, nos referimos a 15 provincias, 168 municipios, más el municipio especial antes mencionado. Este panorama territorial hasta hace algunos años no era similar, y en la actualidad se referencia sin ninguna objeción. Sin embargo, a mediados del 2010, en el archipiélago cubano se implementó una división político-administrativa - que explica la actual organización regional - orientada por el aparato del poder: la Asamblea Nacional del Poder Popular.

Al principio corrieron los rumores que "La Habana campo" sería divida en dos. Aunque esta idea persistió durante algunos años en la primera década del SXXI, se diluía con el tiempo y pocas personas creyeron que se hiciera realidad. En agosto de 2010 se publicaron los primeros titulares en la prensa nacional: "Proponen en pleno del Partido dos nuevas provincias cubanas: Artemisa y Mayabeque" (PCC, 2010), "El parlamento cubano aprueba la creación de dos nuevas provincias" (Asamblea Nacional del Poder Popular, 2010), "Nacerán nuevas provincias con un sentido racional" (Maury, 2010). Aquellos comentarios de los que muchos dudaban fueron realidad, La Habana se dividió y se crearon dos nuevas provincias: Mayabeque y Artemisa.

A pesar de que en la Constitución de la República de Cuba se estipula a La Habana como capital de Cuba (Asamblea Nacional del Poder Popular, 1976), antes de esta división políticoadministrativa existían dos Habanas: la primera, la Ciudad de La Habana, actual territorio de La Habana y capital oficial de Cuba y la segunda provincia, La Habana (más conocida como Habana campo) que se convirtió en las dos nuevas, Mayabeque y Artemisa. Antes de esta división "La Habana campo" funcionaba como una especie de periferia de la ciudad capital. Años precedentes a la división político-administrativa se decía que La Habana cumplía la función de triple capital; primero capital de Cuba, segundo capital de Ciudad de La Habana y tercero capital de La Habana (provincia dividida).

Los argumentos que sustentaron esta decisión fueron la utilización más racional del personal y los recursos, así como la creación de dos regiones en desarrollo con una nueva propuesta de organización político- administrativa. Por consecuencia, Mayabeque y Artemisa surgieron como una especie de laboratorio donde se implementaron las nuevas propuestas de dirección territorial. Los sistemas institucionales fueron reducidos, se crearon las capitales provinciales con arterias de movilización a sus municipios y se propuso un nuevo programa de desarrollo cultural. Finalmente, para varios autores el principal cambio de esta división consistió en la demarcación de las funciones entre las asambleas del poder popular regional y los gobiernos regionales.

Esta división se orientó verticalmente por el Estado cubano, para la cual no se tuvieron en cuenta características socioculturales propias de la región que quizás fueron originarias en siglos anteriores. De un año a otro, los pobladores vieron cambiar en sus documentos de 
identidad la provincia de residencia, su lugar de nacimiento, incluso su puesto de trabajo fue desplazado también. La historia muestra cinco divisiones políticos-administrativas en Cuba desde la colonia. Sin embargo, la "Habana campo" mantuvo su condición de territorio periférico de la capital por más de cuatro siglos, creando lazos identitarios a un nombre, un gentilicio y una memoria colectiva de ser capitalinos. Como nombre territorial se mantenía, pero sus límites geográficos y municipios ya habían sido modificados en la división político administrativa de 1976 (Ley 1304 de 1976). Casualmente, gran parte de los mismos municipios periféricos de la provincia "Habana campo", Pinar del Río y Matanzas reorganizados con esa ley, en solo 35 años sus pobladores vivieron otra división territorial. El nueve de enero de 2011 los solos "habaneros" que respondían a este gentilicio fueron los residentes de la capital.

En la actualidad, para los actores gubernamentales y académicos de las provincias Mayabeque y Artemisa, evocar la división territorial no aporta grandes discusiones, hecho que no refleja la realidad vivida durante los años primeros de su creación. Desde el nombre, hasta la selección del "héroe histórico representativo territorial" provocó discordancias entre ellos. Fue entonces que se evidenció la creación de un discurso de la identidad territorial o regional, que agrupó elementos geográficos, espaciales y patrimoniales, intentando establecer los pilares identitarios que sostenían el proyecto de división territorial.

El discurso sobre la identidad de cada provincia fue creado y sustentado por los medios nacionales y territoriales de comunicación. Como una especie de ave fenix renacieron elementos históricos, paisajes naturales, eventos culturales, sitios emblemáticos; todos con la intención de "fabricar autenticidades" (Bayart, 1996) para la construcción de una nueva identidad territorial. Nuestro estudio se enfoca específicamente en la provincia de Mayabeque. En la pesquisa inicial se detectó que existió una mayor intención mediática en este territorio. Desde su nombre hasta la selección de la capital fue inesperada por todos. Otro aspecto importante fue que, en la división territorial, de cinco municipios de la "Habana Campo" limitantes con la capital, cuatro se otorgaron a Mayabeque.

Mientras que los medios se preocupaban por encontrar elementos para una identidad territorial, la opinión de los pobladores mostró su preocupación por otros aspectos "prácticos del día a día". La dinámica de vida de muchos de ellos se alteró al desplazar las instituciones de la antigua provincia a su actual capital "San José de la Lajas"; fue abandonar la rutina diaria de vida correspondiente a una ciudad cosmopolita con más de 2 millones de habitantes, para adaptarse a la vida en un "pueblo" que recién comenzaba a prepararse como capital provincial.

\section{Identidad y territorio}

El abordaje de la temática de "territorio" se puede encontrar en la historia humana, incluso fuera del ámbito de análisis científico, intentando establecer su definición y razón de ser. Desde su comprensión como área física hasta el entramado simbólico y espacial que se 
construye hoy, incluyendo el entorno virtual, este concepto se entrelaza con diferentes disciplinas de estudio y su construcción merece en la actualidad una mirada transdisciplinaria (Medby, 2018; Rodon and Guinjoan, 2018; Cognard 2011; Vela-Almeida, 2018). Comenzando por la biología, luego la geografía y, seguidamente disciplinas de las ciencias sociales como la sociología, la economía, las ciencias políticas ponen en el centro de las investigaciones el "territorio" y su forma de desarrollo. La noción de territorio suscita cada vez más, análisis que comprendan los fuertes lazos identitarios que desdoblan los individuos alrededor de él y cómo este arraigamiento identitario provoca reacciones encontradas cuando se pone en peligro el "territorio" (Ospina Peralta and Hollenstein 2015; Burgos and Rojas 2013; Re 2016).

La comunidad, así como las personas, desde nuestro nacimiento, juegan un papel que al agregar los espacios físicos y simbólicos contribuyen a la creación del “territorio" (Re, 2016). Es entonces que la identidad constituye un elemento muy importante en la creación de este y todo lo que su significado comprende (Ashley \& Alm, 2016; Belhedi, 2006). Para ello la identificación permitirá un anclaje sólido de las personas a su espacio físico, la delimitación de sus fronteras y la noción de tiempo vivido; creando una noción multidimensional de territorio que permitirá su desarrollo dinámico y sostenible (PNUD, 2014; Sens, 2004).

Al hablar de identidad territorial se debe concebir los debates sobre homogeneidad o heterogeneidad, diferencias y similitudes, presente y pasado, individual y colectivo, o sea, es reconocer su carácter de "doble hélice" (Kaufmann, 2010). Su esencia es la de un concepto dinámico y operativo que responde a objetivos diversos y numerosos; razones que estimulan su utilización para sustentar discursos de la diferencia, pero al mismo tiempo, en otras escalas o puntos de vistas, sostiene la igualdad y la homogeneidad. La identidad territorial presupone el reconocimiento de ciertos significados, de ciertos bienes y ciertas experiencias de vida que se manifiestan para y por los actores territoriales (Ridanpää, 2017). Como consecuencia, se manifiesta en diversos estilos de vida que son el reflejo de las dinámicas territoriales apropiadas por los individuos y las colectividades (Bourdin, 1984). Es así que en los territorios se pueden distinguir quien es de allí o quién es de afuera, los residentes o los extranjeros, y al mismo tiempo, se forman estereotipos ligados, más a la noción de territorios, que de las personas habitantes (Bourdin, 2000).

La "territorialización de la identidad" permite el control político del espacio social (Di Meo, 2007). En espacios urbanos, sobre todo, se constata la concreción organizativa del territorio a partir de las identidades colectivas, elemento utilizado por los poderes hegemónicos para el control dentro de la diversidad regional (Simone, Barile, \& Calabrese, 2018). Aunque parece sencillo el tema de la organización del territorio, estudios muestran que la construcción de las identidades personales no siempre se detiene en las fronteras geográficas (Aquilué \& Roca, 2016; Ashley \& Alm, 2016; Belhedi, 2006; Sofianou, 2016). Cada vez más, con la naturalización de los flujos migratorios que sufren las sociedades actuales, lo individuos construyen sus características identitarias en función de estos movimientos, permitiéndole el desarrollo de su capacidad de resiliencia al transitar entre territorios (Tovar \& Ruíz, 2015; Uribe Castro \& Ramírez Arcila, 2014). 
Para nuestra investigación es importante la idea del territorio a partir de un proceso de construcción social, su resultado se concibe desde la acción social, que de forma concreta y abstracta se apropia de un espacio tanto físico como simbólico (Gonzalez \& Vasquez, 2012; López Santiago \& Barajas Gómez, 2013). Sin embargo, aquí estamos frente a un proceso de re-territorialización, para el cual se deben "redescubrir y revitalizar" significados que sustenten este nuevo territorio a través de construcciones identitarias enunciadas por el discurso político hegemónico (Nora, 2013). A la opinión de Bernard Pecqueur (2000) existen diferencias entre los territorios según su nacimiento: el primero, "el territorio dado", definido por decisión político - administrativa y el segundo, "el territorio construido", que nace del encuentro de actores sociales y se apropian consecuentemente de un espacio geográfico (Pecqueur, 1989, 2000).

Cuando nos encontramos frente a un "territorio dado", memoria e historia se convierten en sus principales aliados para sustentar los discursos identitarios. Para "la invención de la tradición" se buscan esos "significados flotantes o vacíos" (Laclau, 2006), y son reconfigurados y resignificados por el discurso hegemónico. La construcción identitaria del territorio se va apoyar del entrecruzamiento de la memoria - esta como un lazo vivido en presente eterno que se alimenta de recuerdos globales o flotantes, particulares o simbólicos, sensible a todas las transferencias (Nora, 1992)-; y la historia - representación del pasado creada como forma de registro y que es constantemente analizada a partir de evidencias científicas (Nora, 1992)-, ambas, permiten la creación de las identidades primordiales que justifican la pertenencia a un espacio geográfico constituido.

Giménez (2007) explica que el territorio y su relación con la cultura se puede explicar en tres dimensiones: "el territorio constituye por sí mismo un espacio de inscripción de la cultura" (p.129); segunda, puede servir como "área de distribución de instituciones y prácticas culturales espacialmente localizadas" (p.130) y finalmente, el territorio puede ser apropiado subjetivamente como "objeto de representación y de apego afectivo, como símbolo de pertenencia sociocultural” (p.130). La primera dimensión refiere a que la herencia cultural de una región la hace distinta a las demás regiones, impregnando características distintivas de otras. La segunda definición, fuertemente vinculada con la primera, son aquellos "rasgos objetivizados" como las pautas de comportamiento, vestimenta, fiestas populares, entre otros. Finalmente, la última dimensión refiere la interiorización del espacio en su sistema cultural. Esta dimensión nos interesa para el estudio porque el autor explica que la “desterritorialización” no solo se da en términos físicos sino también en términos simbólicos y subjetivos.

Las diferentes aproximaciones teóricas abordadas encuentran una especie de sistematización en la obra Bayart (1996): L'illusion identitaire. El autor explica cómo desde el campo político son utilizados elementos del pasado que a través de un proceso de formalización y ritualización originan nuevas tradiciones delimitando las identidades y fijando la diversidad (Bayart, 1996). El autor para la invención de la tradición propone una serie etapas; la primera "extraversión cultural y transferencia de significado" que refiere relacionar elementos culturales foráneos en el cumplimiento de objetivos autóctonos. La segunda etapa "la 
fabricación de autenticidades", esta pretende preservar la pureza original de los rasgos identitarios de la influencia extranjera. La tercera etapa "la formación de identidades primordiales" que consiste en la búsqueda y revitalización de elementos identitarios particulares y sobre todo históricos, para su inclusión en los discursos actuales. Finalmente, "la formulación política" que comprende el conjunto de medios y estrategias discursivos para anunciar y autentificar esta tradición. Todas estas etapas de forma sistematizada comprenden los diversos abordajes teóricos recurrentes en la literatura, y describen hasta cierta medida lo ocurrido en nuestro caso de estudio.

Para nuestra investigación, las etapas "fabricación de autenticidades" y "la formación de identidades primordiales" agrupan los preceptos teóricos fundamentales para sustentar los estudios y, sobre todo, desde su definición, parten nuestras categorías de análisis. Igualmente es pertinente la noción construcción social, así como anclaje territorial e identitario para el desarrollo dinámico y sostenible, puesto que el slogan principal de creación de la región fue "Mayabeque una provincia en desarrollo".

\section{Metodología}

El análisis de contenido se construyó sobre la base de la teoría de Bayart (1996) la invención de la tradición. Durante el estudio teórico se encontraron algunas coincidencias que permitieron el abordaje de esta teoría como guía referencial para el estudio: primero, la toma de la decisión verticalmente; segundo, la revitalización de la memoria histórica y la historia por diferentes historiadores e investigadores que participaron en el proceso; finalmente, la aparición de todo un discurso político que intentó mostrar la pertinencia de esta decisión desde el punto de vista cultural e identitario. Igualmente se tuvo en cuenta la importancia de comprender la "dinámica territorial" y la "identidad territorial” como piezas claves el desarrollo sostenible y sustentable de la región.

Los resultados alcanzados se obtuvieron a partir del análisis de contenido realizado a un grupo de noticias que mostraban el tratamiento de la identidad territorial de la provincia Mayabeque. Para ello se estableció como período de recopilación el primer año de creación del territorio. Los datos se obtuvieron de los artículos publicados en el periódico regional Mayabeque, en su versión impresa. Este periódico tiene una frecuencia semanal, por lo que se analizaron 51 ejemplares, más tres ediciones especiales.

Los periódicos tenían entre 8 a 10 páginas por ejemplar, por lo que se analizaron todas las secciones que lo componen. Finalmente, partiendo de los postulados teóricos, se construyeron tres temáticas de análisis que abordaban el tema de la identidad territorial: la selección del gentilicio (1 articulo), la identidad territorial a partir de la revitalización de identidades locales ( 6 artículos) y el debate sobre el equipo provincial de béisbol ( 2 artículos). Los análisis de resultados sistematizan los criterios enunciados y que son evidencia de esta intención política. 


\section{Análisis de resultados Ni mayabequenses, ni mayabequinos... nosotros somos habaneros}

Para los cubanos el territorio donde viven es importante, no en la actualidad sino históricamente. Sucesos que se remontan a la época colonial muestran como el regionalismo existente en aquella época, fueron uno de los principales aspectos para el fracaso de la "Guerra de los 10 años" que comenzó en 1868 y terminó en 1878. En la actualidad, estas diferencias identitarias regionales no alcanzan tales extremos, pero cada provincia a través de un slogan popular o institucionalizado identifica a cada habitante del territorio: provincia Camagüey, tierra de camagüeyanos, ciudad de los tinajones donde se habla mejor el español; la provincia de Cienfuegos, la perla del sur y ciudad natal de Benny Moré; la provincia Santiago de Cuba, tierra caliente de santiagueros donde se realizan los mejores carnavales de la isla; por solo citar algunos ejemplos. No obstante, vivir en La Habana y responder al gentilicio de "habaneros" constituye históricamente uno de los principales orgullos identitarios de los pobladores de este territorio y quizás el anhelo de muchos cubanos, es por ello que no interesaba si fuera de "Habana campo" o "Habana capital", lo importante era que todos respondían a "habaneros".

Estas expresiones no sólo dan cuenta del lugar de origen de a quien se refiere, sino que encierran todo un imaginario social que se condensa con una palabra y trae consigo una fuerte carga ideológica y cultural. Hasta cierto punto, el gentilicio empodera al individuo al territorio donde vive, responde a lo que el pueblo cree sobre el origen de su nombre, a lo que forma parte de su cultura (Ridanpää, 2017). Además, el gentilicio funciona como una marca de identidad cultural y fue motivo de discusión entre los historiadores y lingüistas a la hora de su selección (Hertzog \& Ross, 2017).

Se encontró un artículo que explicaba las discusiones en torno a la determinación del gentilicio: mayabequinos y mayabequenses (Rensoli, 2011). El primero sustentado por los historiadores en una posición más conservadora. Para ellos el gentilicio "mayabequinos" respondía a la historia del territorio, al evocar que el nombre de la provincia "Mayabeque" era originario del río principal del territorio y otorgado por los indígenas siboneyes. Además, alegaron que en documentos y registros de siglos anteriores se referían a los pobladores de esta región como "mayabequinos".

El segundo discurso, con principios más innovadores, y sobre todo provenientes de académicos, apostó por "mayabequenses". Los argumentos se sustentaban en la idea de romper con todo vestigio de la región como periferia de la capital, y comenzar a construir nuevos pisos identitarios. Para ellos este gentilicio propiciaba fuerza en la construcción y renovación de la identidad del territorio. Finalmente, se escogió el gentilicio "mayabequenses". No obstante, mientras posturas institucionales discutían por este adjetivo, la población en general deseaba mantener su gentilicio inicial "habaneros" ya que este representaba siglos de memoria e historia local. 


\section{La identidad mayabequense en cuestión}

En la búsqueda realizada, uno de los primeros artículos encontrados fue "La leyenda del Mayabeque" (Asacanio, 2011), su intención, justificar el nombre de la provincia. Mayabeque, según el artículo, es el nombre del río que atraviesa la mayor cantidad de municipios de la región. De norte a sur, el río sirve de fuente de agua para el desarrollo de la agricultura, principal actividad económica de la región. Este nombre es otorgado por las poblaciones indígenas exterminadas con el colonialismo español y que sobrevivió a los nombres introducidos por los españoles con la creación de nuevos pueblos y asentamientos. Los artículos analizados presentaron, como en forma de inventario cultural, todas aquellas características que pueden ser utilizadas en la construcción de la identidad territorial (Antúnes, 2011; Vasquez, 2011). En ellos se describió la región como tierra de historia, que se remonta al holocausto indígena y a la esclavitud, a la reconcentración en el colonialismo español y a la pobreza de sus campesinos. Igualmente reconoció como la principal festividad de la región "las Charangas de Bejucal " en el municipio de Bejucal, así como la festividad de la Santa Bárbara en Güines, y refirió a los platos de Melena del Sur el "mollete" y el plato "la butifarra del congo" en Catalina de Güines como representantes de la cultura tradicional culinaria.

El periodista Omar Felipe Mauri en su artículo: "Mayabeque en una mirada" (Felipe, 2011) centró el análisis desde la principal actividad económica de la región: la producción de azúcar. Por el apogeo de la producción azucarera desde la colonia se atribuyó el surgimiento de las poblaciones de Melena del Sur, San Nicolás de Bari, Nueva Paz, Madruga y Bejucal. Además, resaltó que la región fue pionera en la industria azucarera al introducir la energía hidráulica para mover los molinos, así como el territorio con el primer tramo de línea de tren en todo Latinoamérica (Havane hacia Bejucal, en el año 1838). Seguidamente el autor realizó un recorrido por los principales polos industriales del territorio: el tren eléctrico y el pueblo de Hersey, fábricas de ron en Santa Cruz del Norte y San José de las Lajas y la producción de muebles a partir de desechos de caña de azúcar.

Por otro lado, se encontró un artículo que presentaba y apoyaba a Juan Evangelista Delgado Gonzales como el héroe provincial (Robles, 2011). Juan Evangelista Gonzales Delgado nació en Bejucal, 27 de diciembre de 1868 y se reconoce en la historia de Cuba como un héroe. El dirigió el rescate de los cuerpos muertos de Antonio Maceo y Panchito Gómez Toro de las tropas españolas durante la guerra de independencia (1895-1898); Maceo, sobre todo, es considerado uno de los principales líderes cubanos que protagonizaron las luchas independentistas contra los españoles. Esta acción y otras condecoraciones obtenidas durante las luchas se enfatizaron en el artículo en demostración de su pertinencia como héroe provincial.

"Arte como agua fresca" (Terry, 2011) fue un texto que catalogó a los principales artistas de la región y que formaban parte de la cultura nacional. Autores como Odilo E. Urfé González, 
Antonio Maria Romeu, Tata Guines, Arsenio Rodriguez aparecieron en esta lista como principales exponentes de la cultura regional y orgullo territorial. Finalmente, resaltó la canción titulada "Échale salsita" y creada por Ignacio Piñeiro, la cual ha sido un éxito nacional e internacional y es uno de los principales exponentes del son cubano. El autor también habló sobre la música campesina, especialmente "La Décima campesina" y la tradición de improvisación a lo largo de la historia musical del territorio.

Las tendencias discursivas en todos los artículos evidenciaron la rememoración de lugares históricos y tradiciones culturales de la región. Aproximadamente 16 sitios históricos y 14 tradiciones locales repartidas en los diferentes municipios fueron mencionados en los artículos. Llama la atención como todos estos elementos identitarios antes de la división territorial se encontraban en discursos localistas y bien concentrados en pequeñas poblaciones, sin embargo, en aquel año, fueron resignificados a un nivel regional como representantes de toda la región.

\section{Y entonces, ¿a cuál equipo pertenecemos?}

El deporte es una de las expresiones más importantes del mundo moderno y está en el centro de las identidades nacionales. Los ejemplos de la relación entre el deporte y la construcción de la identidad son comunes, y en Cuba, especialmente a partir de la segunda mitad del siglo XX con la declaración del béisbol como deporte nacional, los equipos de baseball representan a cada provincia. Cada año se celebra el Play Off de Baseball, competencia deportiva que constituye el centro de atención de todos los cubanos.

El equipo de baseball de la antigua Habana tiene sus principales orígenes con la creación del Play Off de Baseball en 1962; o sea 48 años después, los pobladores de la región tuvieron que ver la separación de sus atletas favoritos, adaptarse a un nuevo nombre y pseudónimo y comenzar a construir lazos de identificación entorno al equipo formado por la división territorial. Desde años anteriores para los pobladores de la "Habana campo" era difícil simpatizar con su equipo provincial, porque para ir de compras, estudiar en la universidad, caminar los fines de semana, incluso trabajar, tenías que ir a la capital habanera.

La identificación a un equipo deportivo no solo está dada por sus resultados en el terreno sino por una serie de aspectos influyentes: jugadores, vestuario, tiempo de existencia, tradición familiar, influencia sociocultural, entre otros aspectos que complejizan aún más esta afinidad. Con la división político - administrativa, las personas se encontraron con la encrucijada de escoger entre tres equipos cuál era su favorito: Mayabeque, Artemisa o Industriales (equipo de la capital). Por un lado, vieron a sus jugadores predilectos ubicarse en el equipo contrario (Artemisa), y por otro, establecer lazos de identificación en cuanto a color del uniforme y símbolo provincial se refiere.

"Otro equipo hará su debut en la Serie Nacional de Béisbol. Pero a diferencia del equipo Artemisa, ningún club llamado Mayabeque se registra en la historia del baseball cubano" (Nasser, 2011, p.4). El análisis en los artículos periodísticos estuvo alrededor de las 
principales insignias que representarían al equipo de baseball. El color escogido para el vestuario del equipo y que fue presentado a través de estos artículos fue el marrón y blanco. Según los autores, este color combina perfectamente con la agricultura campesina y la imagen de la región.

La insignia del equipo es "Huracanes de Mayabeque", la hipótesis presentada por los periodistas consistió que esta región cada año es azotada o tiene el riesgo de azote por este tipo de evento natural (Nasser, 2011b). Al paso de los huracanes se evidencia un desastre total, arrasa con todo. Metafóricamente, adoptar esta insignia significaba que el nuevo equipo Mayabeque por su juventud y fuerza, arrasaría en el torneo de baseball alcanzando el título de campeón. El tiempo ha evidenciado que la división y creación de este nuevo equipo no ha sido solamente difícil para los pobladores de Mayabeque, sino internamente desde su creación, hace ya 7 años, no logra salir de los últimos puestos en el certamen deportivo nacional.

\section{Discusión y conclusiones}

La provincia de Mayabeque se identifica, entre otros aspectos, por su gran diversidad y su proximidad a la capital. Este último elemento provoca que los ecos de la ciudad cosmopolita lleguen constantemente a sus pobladores y continúen sentimientos de añoranza por la que una vez fue su capital provincial. Desde su creación, incluso hasta le fecha, se puede argumentar que la identidad territorial ha sido improvisada desde los diferentes discursos. Esta característica coincide con las expuestas por Pecqueur (2000) como estrategia reconocida en diferentes contextos para argumentar el "territorio dado". Igualmente esta idea concuerda con Erika y Franco (2015) cuando expresaron que los procesos de planificación territorial muchas veces responden a las necesidades y proyecciones de gobierno más no a las necesidades colectivas.

La "fabricación de autenticidades" (Bayart, 1996) fue una estrategia corroborada en los análisis de los textos. A través de un inventario de tradiciones, memoria e historia, las identidades locales fueron resaltadas por los autores, en esta ocasión con una perspectiva macro: nivel regional. Igualmente, aquellas figuras representativas de la cultura nacional e internacional fueron "reclamadas" como personas nacidas en la provincia y por ende orgullo de todos los mayabequenses.

Un ejemplo reconocido como fabricación de autenticidades evocado en los artículos fue la celebración a nivel regional (provincial) de la declaración de Melena del Sur (municipio sureño) como "primer territorio libre de analfabetismo en Latinoamérica" el 5 de noviembre de 1961. Sin embargo, esta conmemoración, antes de la división político-administrativa, quedaba completamente olvidada y restringida al marco local del municipio, ya que la fecha principal que se conmemora en todo el país es el 22 de diciembre de 1961 cuando se declaró "Cuba territorio libre analfabetismo". Este hecho en particular es explicado por Nora (2013) cuando dice que la memoria histórica es institucionalizada por un poder hegemónico y se convierte en un "lieu de memoire" para el reforzamiento de la identidad territorial. 
Las opiniones sobre la extensión de la noción de identidad cultural en la provincia son diversas y controvertidas. Los autores consultados otorgan a las identidades un carácter comunitario (étnico, local, regional, nacional, supranacional) y social (género, profesión e individuos), entre otros. Este aspecto coincide con Aquilué \& Roca (2016) al definir la identidad como una categoría dialéctica donde las formas superiores contienen las inferiores. Además, el contexto actual de la provincia muestra la necesidad de introducir en este discurso las relaciones cotidianas de actores, individuos y diferentes grupos sociales, es decir, las prácticas sociales y culturales que se desarrollan en un contexto determinado, que podría estar en la comunidad o en un área mucho más grande que la ciudad. Con esto, podemos decir que la identidad también se construye a partir de prácticas sociales para satisfacer las necesidades. Generalmente, desarrollamos grupos e individuos "identificados" en nuestras vidas diarias.

Las iniciativas artificiales u orientadas verticalmente para la construcción de un territorio requieren un esfuerzo mayor para el fomento de la imagen local y el sentido de pertenencia al lugar. El análisis de contenido mostró que la identidad territorial, incluso cuando se mantiene el mismo espacio geográfico, se construye a partir de diferentes aspectos socioculturales, de ahí la necesidad de crear discursos que refuercen la noción de territorio. La práctica de estas iniciativas concuerda con el concepto de pertenencia sociocultural de Giménez (2007) donde "la dimensión territorial caracteriza de modo relevante la estructura misma de la colectividad y de los roles asumidos por los actores" (p. 132). En la actualidad se constatan todavía intentos mediáticos por justificar lo que se realizó hace 7 años, el peso de la historia habla por si solo, durante casi cuatro siglos los pobladores se acostaron siendo habaneros y de un día al otro, casi sin entenderlo e incluso asimilarlo, se levantaron siendo mayabequenses. 


\section{Referencias bibliográficas}

Antúnes, R. (2011, January). A orillas del Mayabeque. Mayabeque, p. 6.

Aquilué, I., \& Roca, E. (2016). Urban development after the Bosnian War: The division of Sarajevo's territory and the construction of East Sarajevo. JCIT, 58, 152-163. https://doi.org/10.1016/j.cities.2016.05.008

Asamblea Nacional del Poder Popular. (2010). Parlamento cubano aprueba creación de dos nuevas provincias. Cubadebate.

Ascanio, N. (2011, January). La leyenda del Mayabeque. Mayabeque, p. 5.

Bayart, J.-F. (1996). L'illusion identitaire. Paris: Fayard.

Belhedi, A. (2006). Territoires, appartenance et identification. Quelques réflexions partir du cas tunisien. Espace Geographique, 35(4), 310-316. https://doi.org/10.3917/eg.354.0310

Bourdin, A. (1984). Le patrimoine réinventé (PUF). Paris.

Bourdin, A. (2000). La question locale (PUF). Paris.

Burgos, R. M. B., \& Rojas, J. C. G. (2013). Son huasteco e identidad regional. Investigaciones Geográficas, 80(80), 86-97. https://doi.org/10.1016/S01884611(13)72753-5

Cognard, F. (2011). Une approche géohistorique de l'identité territoriale néo-zélandaise. L'Information Géographique, 75(2), 6. https://doi.org/10.3917/lig.752.0006

Di Meo, G. (2007). Identités et territoires: des rapports accentués en milieu urbain? Metropopole, 1, 69-94.

Erika, L., \& Franco, O. (2015). La construcción de la pertenencia socioterritorial : el caso de Jurica, un pueblo al que le llegó la ciudad. Espiral, Estudios Sobre Estado Y Sociedad, XXII(62), 141-170.

Felipe, O. (2011, June). Mayabeque en una mirada. Mayabeque, p. 7.

Giménez, G. (2007). Estudio sobre la cultura y las identidades sociales. (C. N. para la C. y las A. / I. C. de Cultura, Ed.) (Primera). Ciudad México: Consejo Nacional para la cultura y las Artes. 
Gonzalez, R. R., \& Vasquez, J. A. ldrey. (2012). De l'identité territoriale au développement local par la restauration et les productions localisées (l'exemple du Cocido de Lalín en Galice). Norois, 3(224), 1-15.

Hertzog, W., \& Ross, N. (2017). Language ideology , space , and place-based identity formation among the Tzotzil Maya of Chiapas, Mexico. Language \& Communication, 59, 1-15. https://doi.org/10.1016/j.langsci.2016.06.001

Johnson, A., \& Alm, L. (2016). Western regional identity and urban development policy: The view from professional planners. The Social Science Journal, 53(2), 143-155. https://doi.org/10.1016/j.soscij.2015.12.001

Kaufmann, J.-C. (2010). L'invention de soi : une théorie de l'identité. Paris: Armand Colin.

Laclau, E. (2006). La razon populista (Fondo de C). Buenos Aires.

López Santiago, N., \& Barajas Gómez, V. B. (2013). Identidad y desarrollo: el caso de la subregión alta mixe de oaxaca. Península, 8(2), 9-37. https://doi.org/10.1016/S18705766(13)71794-X

Maury, M. (2010, September). Nacerán nuevas provincias con un sentido racional. Cubadebate.

Medby, I. A. (2018). Articulating state identity: "Peopling" the Arctic state. Political Geography, 62, 116-125. https://doi.org/10.1016/j.polgeo.2017.10.008

Nasser, A. (2011a, February). Integrado equipo de Mayabeque a la Serie Nacional de Béisbol. Mayabeque, p. 4.

Nasser, A. (2011b, February). Mayabeque debuta con su equipo de baseball. Mayabeque, p. 4.

Nora, P. (1992). Les lieux de mémoire (Gallimard). Paris.

Nora, P. (2013). La culture du passé. (Gallimard, Ed.). Paris.

PCC, P. C. de C. (2010). Proponen en Pleno del Partido dos nuevas provincias cubanas: Artemisa y Mayabeque. Cubadebate.

Pecqueur, B. (2000). Le développement local : pour une économie des territoires (2e éd. rev). Paris. 
Peralta, P. O., \& Hollenstein, P. (2015). Territorial Coalitions and Rural Dynamics in Ecuador . Why History Matters. World Development, 73, 85-95. https://doi.org/10.1016/j.worlddev.2014.10.026

PNUD, P. de las N. U. para el D. (2014). ¿ Cultura y Desarrollo ? ¿ Desarrollo y Cultura? Cuadernos Desarrollo, 177.

Re, A. (2016). San Gregorio Magno, Viaje Entre Memorias E Identidad. Estudio Socioantropológico De Una Comunidad Del Mezzogiorno Italiano. Península, 11(2), 85-100. https://doi.org/10.1016/j.pnsla.2015.04.001

Rensoli, J. R. (2011, May). Mayabeque experimenta su identidad. Mayabeque, p. 7.

Ridanpää, J. (2017). Narrativizing (and laughing) spatial identities together in Meänkielispeaking minorities. Geoforum. https://doi.org/10.1016/j.geoforum.2017.05.003

Robles, A. (2011, March). Patriota insigne y escudo de mayabeque. Mayabeque, p. 6.

Rodon, T., \& Guinjoan, M. (2018). When the context matters : Identity, secession and the spatial dimension in Catalonia *. Political Geography, 63, 75-87. https://doi.org/10.1016/j.polgeo.2018.01.004

Sens, A. (2004). ¿Cómo importa la cultura en el desarrollo? Letras Libres, (54), 23-30.

Simone, C., Barile, S., \& Calabrese, M. (2018). Managing territory and its complexity: a decision-making model based on the viable system approach (VsA). Land Use Policy, 72(July 2017), 493-502. https://doi.org/https://doi.org/10.1016/j.landusepol.2017.12.070

Terry, M. (2011, June). Arte como agua fresca. Mayabeque, p. 7.

Torres Cueva, E., \& Vega Loyola, O. (2001). Historia de Cuba (1492-1898) Formación y liberación de la Nación (Primera). La Habana: Pueblo y Educación.

Tovar, M. R., \& Ruíz, D. T. M. (2015). La Configuración Identitaria En Los Territorios De Migrantes Internacionales. Península, 10(2), 117-133. https://doi.org/10.1016/j.pnsla.2015.08.006

Uribe Castro, H., \& Ramírez Arcila, A. F. (2014). Sentidos de lugar y movimiento social: indígenas de Toribío y defensa de su territorio en Colombia. Latinoamérica. Revista de Estudios Latinoamericanos, 58, 9-31. https://doi.org/10.1016/S16658574(14)70099-7

Vasquez, R. (2011, April). Rasgos distintivos de mayabeque. Mayabeque, p. 7. 
InterSedes, $\mathbf{N}^{\circ}$ 41. Vol XX (2019). ISSN 2215-2458

Vela-almeida, D. (2018). Territorial partitions, the production of mining territory and the building of a post-neoliberal and plurinational state in Ecuador. Political Geography, 62, 126-136. https://doi.org/10.1016/j.polgeo.2017.10.011 